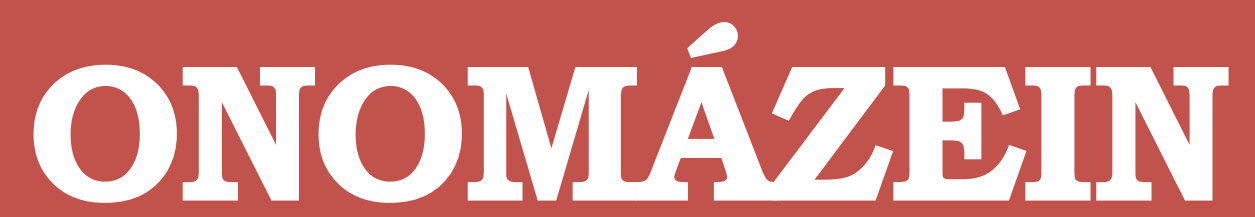

Revista de lingüística, filología y traducción
PONTIFICIA UNIVERSIDAD CATÓLICA DE CHILE FACUITAD DE LETRAS

\title{
Variación y adaptabilidad: los tratamientos en la comunicación por SMS en español bonaerense
}

Variation and adaptability: forms of address in SMS communication in Buenos Aires Spanish

Número especial

- IV -

Apelación en el discurso digital

2018
ONOMÁZEIN - Número especial IV: Apelación en el discurso digital (octubre de 2018): 01-25 DOI: 10.7764/onomazein.add.04

ISSN: 0718-5758

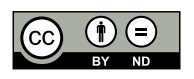

Lucía Cantamutto: Departamento de Humanidades, Universidad Nacional del Sur / CONICET. Argentina. | Correo electrónico: luciacantamutto@gmail.com

Fecha de recepción: noviembre de 2016 Fecha de aceptación: noviembre de 2017 


\section{Resumen}

El artículo analiza un corpus de SMS pertenecientes a la variedad del español bonaerense, poniendo el foco de atención en las fórmulas de tratamiento, a la luz de las categorías descriptas como un estilo económico que está "marcado por la acumulación de recursos expresivos" (Vela Delfa, 2005: 670) y que trata de no ser ambiguo (Cantamutto, 2014). La hipótesis principal sostiene que en las elecciones lingüísticas realizadas por los hablantes para la construcción discursiva de las interacciones por SMS, operan recursos y estrategias propios de la comunicación escrita mediada por teléfono móvil además de cuestiones identitarias vinculadas a la edad y género. Como se evidencia en los resultados de investigaciones previas sobre el corpus SMS-CEBo (Cantamutto, 2012-2016), las formas nominales y los pronombres personales, innecesarios para la construcción sintáctica, aparecen de manera expresa, debido a que permiten construir la fuerza ilocutiva del mensaje así como favorecer la personalización y focalización del destinatario, funcionando como elementos afiliativos en la interacción y que canalizan la cortesía. En la comunicación por SMS, la ausencia de fórmulas nominales de tratamiento causa respuestas no preferidas.

Palabras clave: discurso digital; mensajes de texto; fórmulas de tratamiento; comunicación porSMS.

\section{Abstract}

This article resumes the analysis of SMS in the Spanish dialectal variety of Buenos Aires, focalizing around the address forms, in light of the categories described within an economic style which "is marked by the accumulation of expressive resources" (Vela Delfa, 2005: 670) and tries to not be ambiguous (Cantamutto, 2014). The hypothesis explains the linguistic choices made by speakers for the discursive construction of SMS interactions, related to the use of resources and strategies specific to written communication mediated by mobile telephony, along with identity features denoting age and gender. As evidenced by previous analysis of the corpus SMS-CEBo (Cantamutto, 2012-2016), nominal forms and personal pronouns that are unnecessary for syntactic construction are expressed, but those elements are aimed at constructing the illocutionary force of the message and at personalizing and focusing on the addressee. Address forms, along with other expressive features, serve as affiliative traits among interacting and canalize politeness. In SMS communication, the absence of nominal forms of treatment causes adverse responses.

Keywords: digital discourse; text messages; address forms; SMS communication. 


\section{Introducción}

El intercambio de mensajes de texto (SMS1) a través de teléfonos móviles ha sido uno de los objetos de atención de los estudios sobre comunicación digital: en épocas tempranas, atendiendo a sus características ortotipográficas que derivaron en posturas divergentes en torno al "lenguaje SMS" (Galán Rodriguéz, 2002 y 2003; Maris y Henin, 2002; Avedaño, 2007; Álvarez Gonzaga, 2010; Cantamutto, 2012; Cougnon, 2015) para, luego, observar las condiciones de enunciación, la conformación discursiva y aspectos relativos a su dinámica interaccional (Tagg, 2009; Tjora, 2011; Bernicot y otros, 2012; Bucher, 2015; Morel y otros, 2012; Cantamutto, 2015b, 2016). En esta última línea, nuestro interés actual son unos elementos particulares que tienen gran prominencia en los intercambios de textos breves: las funciones que cumplen las fórmulas de tratamiento nominales en función vocativa y el lugar que ocupan dentro de las formas de apelación.

Un conjunto de trabajos que se constituyen como antecedentes de nuestra investigación son aquellos que abordan la interacción digital desde la perspectiva del análisis de la conversación. Esta línea, inaugurada por Susan Herring (1996), se interesa por delimitar la estructura discursiva de los mensajes electrónicos, y la organización y coherencia de la interacción digital. Diferentes autores analizan la funcionalidad de las unidades de análisis de esta teoría para diferentes plataformas: por ejemplo, para el chat (Noblía, 1999; Neuage, 2005; Vela Delfa, 2014), para el correo electrónico (Vela Delfa, 2005; López Alonso, 2006), para la MI (Vela Delfa y Jiménez Gómez, 2011; Vela Delfa, 2014), para la comunicación por SMS (Cantamutto, 2013b; Xue, Su y Jeong, 2016) y, recientemente, para la comunicación por Whatsapp (Alcántara-Plá, 2014; Martín Gascueña, 2016). En términos generales, se detecta un movimiento pendular de apropiación de las categorías del análisis de la conversación cara-a-cara para expresar su insuficiencia al momento de ser aplicadas a la interacción digital, principalmente, por la dificultad para establecer los pares de adyacencia. En particular, los estudios sobre los SMS, que surgen después del año 2000 con algunas excepciones en los finales de la década de los 90 (Cougnon y Fairon, 2012: 157), adolecen de estudios longitudinales y con grandes corpus en el español.

Dentro de los géneros del discurso digital, el texto breve es un tipo discursivo cuyas principales características son su carácter monomodal y verbal (vs. otros géneros más multimodales), son breves (vs. otros tipos más extensos), tienden a la continuidad comunicativa, son permanentes, unidireccionales, privados e íntimos (véase Cantamutto y Vela Delfa, 2016). El SMS es una de las posibilidades interaccionales con las que cuenta el usuario hablante, como nodo (Yus, 2010), en su teléfono móvil y que, en la actualidad, ha caído en desuso a partir del

1 SMS es una sigla en inglés que corresponde a "short message service". Usamos esta sigla dado que es la de empleo más generalizado en los estudios sobre el tema. Sin embargo, no existe aún consenso en la forma de nombrar este objeto de estudio (véase Panckhrust, 2009: 35; Tagg, 2009: 1). 
predominio de las plataformas de mensajería instantánea (WhastApp, Line, Telegram o Hangout). La interacción por mensajería instantánea (MI) se asemeja, en parte, al intercambio de SMS (Calero Vaquera, 2014; Yus, 2016), aunque estos tienen su especificidad, que radica en las limitaciones que presenta (cantidad de caracteres, bajo nivel de multimodalidad y marcas de retroalimentación) y en el uso de una red de trasmisión de datos diferente (razón por la cual aún hoy, ante problemas de conectividad a internet, los usuarios intercambian SMS con propósitos concretos).

Guiados por los hallazgos previos de nuestra investigación² que cristalizan las tres estrategias propias de la comunicación por SMS: economía, claridad y expresividad (Cantamutto, 2017), nos es posible inferir que aquellos recursos que permitan al hablante mayor expresividad y que, a su vez, requieran de menor cantidad de elementos o esfuerzo (es decir, se identifiquen con el principio de economía) tendrán más recurrencia dentro del discurso de Ios SMS. Tal es el caso, por ejemplo, de las estrategias textuales (Yus, 2010) como el uso de abreviaciones, creación léxica y repetición de letras y/o signos de puntuación (Cantamutto, 2012, 2014, 2016, 2017).

Advertimos, sin embargo, que la economía lingüística no es equiparable con el límite de caracteres, sino que la tendencia a la optimización de los recursos lingüísticos es la que favorece el uso de estrategias más eficaces (ver Paredes Duarte, 2008). En palabras de Vela Delfa, "el carácter económico del estilo electrónico no sólo se manifiesta en el rango de su organización textual y sintáctica sino en niveles más concretos, como la propia selección léxica o su realización ortográfica” (2005: 670). En la mayoría de los SMS de nuestro corpus, la economía - que muchas veces no favorece la gestión de los vínculos-convive con alguno de los posibles recursos de expresividad (Cantamutto, 2012) que estimulan la relación interpersonal (Kerbrat-Orecchioni, 1996).

En esta línea, la hipótesis de nuestra investigación es que, en la construcción discursiva de las interacciones por SMS, las elecciones lingüísticas que realizan los hablantes están atravesadas por recursos y estrategias propios de esta comunicación, cuestiones identitarias vinculadas a los grupos etarios y al género de los hablantes implicados, y fenómenos relativos al uso de la lengua en contexto. A partir de esta conjetura general, nuestro objetivo, en el presente artículo, es definir y caracterizar, dentro del sistema de las fórmulas de tratamiento

2 Este trabajo forma parte de una investigación mayor, en curso, abocada al estudio de los aspectos pragmáticos de la comunicación por SMS. Este estudio es financiado por una beca doctoral de CONICET, dirigido por la Dra. Elizabeth. M. Rigatuso, y se realiza en el Centro de Estudios Lingüísticos "Dra. Fontanella de Weinberg" (Universidad Nacional del Sur), en el marco del proyecto de investigación "Estilo(s) comunicativo(s) y variación pragmática en la interacción verbal del español bonaerense: construcción de identidades, valores y creencias", subsidiado por la Secretaría General Ciencia y Técnica de la Universidad Nacional del Sur. 
del español bonaerense (sección 4.1), las formas nominales y pronominales presentes en la comunicación por SMS según variables contextuales y sociodemográficas, y ponerlas en relación con el lugar que ocupan dentro del conjunto de fórmulas de apelación (sección 4.2).

El estudio de las fórmulas de tratamiento empleadas en la interacción ofrece información respecto a los mecanismos de regulación de la distancia social y reflejan las relaciones de los interactuantes (Brown y Gilman, 1960). En estas páginas, observaremos la variación pragmática intralingüística en el uso de los vocativos - dentro del conjunto de formas apelativas - en la variedad dialectal de español bonaerense. Se comprueba que, en el intercambio de SMS, el empleo de formas vocativas opera como un eficaz recurso para personalizar el mensaje y como estrategia para conducir las estrategias propias de la cortesía verbal (Cantamutto, 2017).

Este artículo se estructura del siguiente modo. En primer lugar, se presenta la metodología de recolección de los SMS utilizada en el análisis (sección 2), luego se presenta el marco teórico (sección 3). A continuación, se ofrece, primero, una aproximación al sistema de las fórmulas de tratamiento en los SMS y, posteriormente, el análisis cualitativo y cuantitativo del uso de los vocativos y su frecuencia (sección 4). En la última sección, se exhibe un resumen de los hallazgos junto con la discusión de los mismos.

\section{Metodología}

Los datos analizados provienen del corpus SMS-CEBo - Corpus del español bonaerense de mensajes de texto-, recolectado en la ciudad de Bahía Blanca entre 2008 y 2016, actualmente integrado al proyecto CoDiCE —Comunicación Digital: Corpus del Español- (Vela Delfa y Cantamutto, 2015a, 2015b). Las muestras de lenguas, pertenecientes a la variedad del español bonaerense, fueron recogidas a través de diferentes técnicas. Por un lado, la transcripción manuscrita en plantillas en papel y, en una etapa posterior, mediante plantillas online (enviadas por correo electrónico y redes sociales), obtenidas por colaboradores voluntarios que transcriben mensajes recibidos y enviados a partir de instrucciones precisas para realizarlo. Por otro, transcripciones, realizadas por la propia autora, de SMS extraídos de teléfonos de familiares y amigos que cedieron sus mensajes de manera voluntaria. En las tres técnicas se solicitó la firma de un consentimiento informado como medida previa a la disposición de los datos $^{3}$. A fin de salvaguardar la imagen de los colaboradores en estos intercambios privados, se modificaron todos los nombres propios y toda otra información privada que pudiera facilitar la identificación de los participantes.

3 Para más información sobre el modo de constitución del corpus, véase Cantamutto (2013a, 2012, 2016, 2017). 
La metodología ha sido descripta en profundidad en Cantamutto (2013a) y sigue los protocolos de CoDiCE (Vela Delfa y Cantamutto, 2015a, 2015b). Además, en las diferentes etapas de recolección se siguieron las técnicas propuestas por Tagg (2009), al trabajar con redes sociales de amigos y familiares. Los datos de SMS-CEBo atienden a las variables sociolingüísticas de género, edad y grupo socioeducativo, así como también el ámbito al que pertenecen los intercambios (principalmente, laboral, familiar y de las relaciones sociales) y que resultan operativos para el abordaje y sistematización de la dinámica de las fórmulas de tratamiento (ver, al respecto, Rigatuso, 1992). En la mayoría de los casos, se recogió información complementaria sobre el tipo de teléfono y sistema de escritura utilizado. El análisis de los datos combina tanto una metodología cualitativa como cuantitativa. Para el procesamiento cuantitativo de los datos, se utilizaron herramientas de análisis disponibles en CoDiCE y el paquete estadístico SPPS.

Al consignar los ejemplos, utilizamos un protocolo diseñado tempranamente en nuestra investigación que facilita la lectura de los datos, creando grupos de estudio. De este modo, los datos de SMS-CEBo se citan de la siguiente manera: primero, femenino (F) o masculino (M); luego, el grupo etario en el que se inscribe: menor a 20 años (1), entre 21-35 años (2), entre 36 y 50 años (3), entre 51 y 65 años (4) y más de 65 años (5); tercero, el grupo escolar alto/medio (A) o bajo (B). Antes de introducir el enunciado, se consigna entre paréntesis la clave numérica del SMS dentro del corpus seguida de un guión bajo y el año de recolección del mensaje4 ${ }^{4}$. Así, por ejemplo, "F-3-A (13_2011)" es mensaje de una mujer de entre 36 y 50 años de nivel socioeducativo alto o medio, cuya clave de identificación es el número 13 y que fue recolectado en 2011.

\section{Abordaje teórico}

Desde una perspectiva sociolingüística, con aportaciones del análisis del discurso y de la pragmática, el estudio de las fórmulas de tratamiento ha sido objeto de numerosas investigaciones en el ámbito hispánico (Hummel, Kluge y Vázquez, 2010), para las diferentes variedades del español de Argentina y, en particular, en el español bonaerense — desde una perspectiva histórica y sincrónica- (Rigatuso, 1987, 2003, 2009, 2014, entre otros). Las fórmulas de tratamiento pueden definirse como el repertorio de elementos (nominales y pronominales) que los hablantes de una determinada comunidad utilizan con una triple funcionalidad: modo

4 Una de las variables que opera en los rasgos ortotipográficos de los SMS es el tipo de teléfono, teclado y sistema de escritura empleado por el usuario hablante. Al recuperar la fecha de recolección podemos suponer (en caso de que no haya sido relevado junto con la información sociolingüística del hablante) en qué estadio de evolución de los teléfonos se estaba. En tal sentido, se establece una correlación entre las variables sociodemográficas y el grado de acceso y penetración de la tecnología. 
vocativo (para dirigirse al destinatario), modo referencial (autorreferencial y alorreferencial) y de designación general (Rigatuso, 2000: 296-297). Al interior de los enunciados comprende el sistema nominal, pronominal y las formas verbales correspondientes.

Basados en la propuesta pionera de Brown y Gilman (1960), muchos estudios han coincidido en que el sistema de fórmulas de tratamiento de una comunidad permite verificar la relación entre los interlocutores a partir de la simetría/asimetría existente entre dichos participantes, según parámetros como la proximidad/distancia, informalidad/formalidad, confianza/respeto, solidaridad/poder (Calsamiglia Blancafort y Tusón Valls, 2002: 141). Esta línea argumental sostiene la existencia de dos ejes: un eje horizontal o de solidaridad, cuya base es la relación afectiva y/o cercana entre los interlocutores y que permite, por ejemplo, el tuteo recíproco o el usted recíproco, en relaciones más formales; y un eje vertical o de poder, donde se evidencian las diferencias sociales (basadas en los roles y en la situación comunicativa particular) y que no favorece el tuteo recíproco (para una discusión, véase Ghezzi y Sampedro Mella, 2015).

Nuestro estudio se centra en uno de los elementos periféricos de la estructura oracional, desde la perspectiva gramatical, aunque no en la perspectiva pragmática, como lo son las fórmulas de tratamiento en función vocativa; el vocativo, según propone Di Tullio (2010: 142), "sirve para nombrar al destinatario a quien se dirige el enunciado (...). Generalmente ocupa la posición inicial aunque puede hallarse en otras posiciones (...). No mantiene relación sintáctica con ninguno de los componentes de la oración, por lo que puede omitirse sin consecuencia”. Es decir, su función es principalmente apelativa-fática y, en la estructura del enunciado, puede adquirir valores diferentes según ámbito de uso y en los diferentes grupos sociolingüísticos.

En la variedad dialectal del español bonaerense, las formas nominales y, en algunos casos particulares, las formas pronominales de segunda persona son las que pueden constituirse como formas vocativas (Rigatuso, 2007: 82). Tal como propone Rigatuso (2007: 81), en su uso, este elemento pragmático posee dos funciones principales (cfr. Leech, 1999):

a) Término de llamado de atención.

b) Elemento de focalización interaccional para la expresión de cortesía.

El primer uso suele observarse dentro de la preparación conversacional (Rigatuso, 1987; Van Dijk, 2000), mientras que el segundo puede darse en cualquiera de las fases de la conversación. De todas maneras, la polifuncionalidad del vocativo no se circunscribe a estas dos funciones (Rigatuso, 2000, 2009), ya que, como en la comunicación cara-a-cara, en el intercambio epistolar o telefónico se refuerzan en cada elemento múltiples funciones orientadas a la expresividad del enunciado. Aspecto que maximiza el valor que adquiere dentro de los recursos y estrategias propios de la comunicación por SMS. 
Siguiendo las aportaciones de la teoría de la cortesía (Brown y Levinson, 1987), dentro de los grupos de estrategias que señalan los autores, las fórmulas de tratamiento se inscriben en línea con las que están orientadas a la identidad del otro, pero estas se subordinan a las metas comunicativas (véase Zimmerman, 2005: 246-247). En los SMS se verifican elecciones lingüísticas que atienden a las relaciones interpersonales, particularmente en la elección de formas lingüísticas que tiendan a ubicar a los interactuantes en el polo de la solidaridad. Es decir, durante la interacción se desarrollan estrategias y recursos pragmáticos vinculados a la gestión interrelacional (Spencer-Oatey, 2000).

De esta manera, coincidimos con Rigatuso (2007: 84) sobre las posibilidades que se le abren al hablante al elegir ${ }^{5}$

la voz o el conjunto de voces que en forma más apropiada responda a su intención comunicativa, realizando una apelación inicial como llamado de atención, concretando un acto de saludo, ponderando, minimizando el mensaje, agradeciendo, solicitando algo, elogiando, profiriendo insultos, marcando un perfil de personalidad, destacando habilidades intelectuales o físicas, ofreciendo una elevación del destinatario para expresar cortesía o descortesía, integrando — desde una perspectiva de cortesía afiliativa - al o los destinatarios del vocativo al grupo del productor del discurso o recortando su identidad, entre otras funciones comunicativas ${ }^{6}$.

En tal sentido, los vocativos cumplen una función para la implementación tanto de la cortesía normativa como de la cortesía estratégica (Bravo, 2001; Briz, 2004; Rigatuso, 2009). Como señala Alonso-Cortés (1999: 4037), “[a] I dirigirse al oyente empleando el nombre propio (o un nombre común caracterizador) el hablante puede mostrar una actitud de cortesía. Esta máxima de cortesía establece que en el intercambio verbal el hablante puede dirigirse al oyente manifestando una actitud de afecto, de cortesía, una formal (o negativa) y otra informal (o positiva)".

5 A pesar de la importancia de las fórmulas de tratamiento en la interacción, no existe aún suficiente literatura sobre su importancia pragmática ni su función estratégica. Como Rigatuso sugiere, los estudios sobre los tratamientos se centran en cómo funcionan dentro de determinadas díadas (por ejemplo, empleador/ empleado), para expresar el grado de conocimiento entre los interactuantes, para la toma de turnos, etc.

6 En otro artículo, Rigatuso (1987: 162, nota 4) señala los siguientes papeles que pueden desempeñar Ios tratamientos:

a) Expresión del conocimiento de los participantes respecto al papel y estado que poseen en un contexto concreto de uso lingüístico.

b) Manifestación de deferencia explícita.

c) Asignación de turnos en la conversación.

d) Interrupción momentánea de la pauta de uso vigente en una determinada relación como expresión lingüística de exaltación emocional pasajera en situaciones de intensidad afectiva, severidad, enojo, ironía, etc. 
La propuesta de Zimmerman (2005) en su análisis de los jóvenes esgrime una triple funcionalidad de las interacciones que abarcan las metas ilocutivas, las metas concretas y las metas de identidad/imagen. Estas estrategias de (des)cortesía favorecen la construcción de "la identidad/imagen (face) del ego o la de constituir y respetar la identidad/imagen (face) del otro/ alter. Muchas veces están destinadas a las dos al mismo tiempo, ya que ciertas identidades/ imágenes son complementarias" (Zimmerman, 2005: 245-46). En este sentido, Kaul de Marlangeon (2008) entiende la descortesía verbal no como un fenómeno marcado o periférico dentro de las manifestaciones de los hablantes, sino que suele ser parte del repertorio de estrategias que tienen los hablantes para manifestar su adscripción o rechazo a determinado grupo.

Integramos a este marco teórico la perspectiva pragmática propuesta por Verschueren (2002). Este autor propone conceptualizar los usos lingüísticos a través de propiedades del lenguaje que modelan las elecciones de los hablantes: la variabilidad, la negociabilidad y la adaptabilidad. En palabras de Verschueren (2002: 110), "el uso del lenguaje debe consistir en la continua elección lingüística, consciente o inconsciente, por razones internas (p. ej. estructurales) y/o externas" (cursivas en el original). El autor define la variabilidad como "la gama de posibilidades dentro de las cuales se puede seleccionar" (ibíd.: 115), siempre mutable, que se negocia dentro de la misma interacción. Consecuentemente, la negociabilidad es la causa de que "las elecciones no se hagan mecánicamente o según unas reglas estrictas o una relación de forma-función fijas, sino basadas en principios y estrategias altamente flexibles" (ibíd.: 116) y está indeterminada tanto por el emisor como por parte del intérprete. Debe ser, por tanto, permanentemente renegociada. Por último, la adaptabilidad es la capacidad de "hacer elecciones lingüísticas dentro de una gama de posibilidades variables de modo que se acerquen a la satisfacción de sus necesidades comunicativas” (ibíd.: 119). Esta propiedad no es unidireccional: como las otras dos propiedades es dinámica y coyuntural y, por tanto, objeto de reorganizaciones constantes.

\section{El sistema de tratamiento en los SMS 4.1. Formas pronominales y verbales}

El sistema pronominal actual del español bonaerense está caracterizado por la confluencia de dos formas pronominales (tú-vos / usted), que se verifican en la interacción cara-a-cara y que se distribuyen, generalmente, siendo el primero un tratamiento de confianza y el segundo un tratamiento de respeto. En general, son pocos los intercambios de SMS que utilizan la forma de respeto. En primer lugar, una conjetura que proponemos para explicar esta baja presencia de las formas en usted es que se deriva, inicialmente, del avance del vos en detrimento del usted en el español bonaerense (Rigatuso, 2008, 2014, entre otros) y, en segundo término, del tipo de intercambios que se realizan por SMS: basados en una relación de confianza donde predominan intercambios de la esfera privada. Sin embargo, cuando los valores se enfocan en los ámbitos de uso, la forma usted aparece en el ámbito laboral pero con una moderada 
presencia. El uso de usted aparece como una forma de respeto utilizada por personas que están en el polo de mayor poder pero que, sin embargo, tienen menor edad que el interlocutor. En algunos casos, el tratamiento de usted se mantiene (ejemplo 1), mientras que en otros se produce una asimilación hacia un eje horizontal por parte del empleador (ejemplo 2), por parte del empleado (ejemplo 3) o incongruencias dentro de un mismo mensaje (ejemplo 4).

Ejemplo 1 (usted - usted):

Ámbito de uso: laboral. Contexto: una joven envía un mensaje a una señora mayor que le ayuda en las tareas domésticas, para consultarle si tiene disponibilidad para planchar.

F-2-A $\rightarrow$ F-5-B Buen dia Elsa, como esta? Le escribo para saber si podría plancharnos una

(6197_2015) tanda de ropa. Saludos, María

F-5-B $\rightarrow$ F-2-A Hola si traigamela como las 10 puede saludos

(6198_2015)

Ejemplo 2 (usted - vos):

Ámbito de uso: laboral. Contexto: un joven (Juan) que está buscando trabajo escribe a la persona encargada de tomar gente en una empresa (cuyo apellido es Raitu) para consultarle si hay puestos de empleo disponibles.

M-2-B $\rightarrow$ M-2-A Hola raitu queria saber si ai [hay] una posibilidad.de trabajo..soi [soy] juan (3605_2014) porcel usted me dijo que lo yame [llame].

M-2-A $\rightarrow$ M-2-B Juan, como estas 7

(3606_2014)

M-2-B $\rightarrow$ M-2-A Biem [bien] habra [habrá] posibilidad de trabajo.perdon si lo molesto.

(3607_2014)

$\mathrm{M}-2-\mathrm{A} \rightarrow \mathrm{M}-2-\mathrm{B} \quad J$ Jan, como estas? Estoy con varios preocupacionales ya hechos, yo te aviso (3608_2014) cuando este necesitando mas personal

M-2-B $\rightarrow$ M-2-A Dale..yo le avisaba porque usted me avia [había] dicho que lo yamara [llama(3609_2014) ra]..bien ahora una changua [changa ] tengo..listo lo yamo o usted me avisa...

$M-2-A \rightarrow M-2-B \quad$ Ok. Yo te aviso

(3610_2014):

Ejemplo 3 (vos - usted):

Ámbito de uso: laboral. Contexto: un empleado le escribe a su jefe para preguntarle si tiene que trabajar el día que le corresponde su franco.

7 Por el mensaje 3608, es posible suponer que el hablante envió accidentalmente este SMS y que no era su intención establecer una pregunta típica de la apertura conversacional. 
M-4-B $\rightarrow$ M-2-A Hola mañana tengo franco no te olvide[s] va a quedar un[o] solo contesté (4760_2014): soy paue

M-2-A $\rightarrow$ M-4-B Si, habia [había] pensando en eso. Usted puede quedarse mañana, le paso las (4761_2014): $\quad$ horas como extras

Ejemplo 4 (vos):

Ámbito de uso: comercial. Contexto: una joven, empleada de una óptica, le escribe a Román para avisarle que están listos sus anteojos.

F-3-A $\rightarrow$ M-2-A Hola Román! Soy Paula de la óptima [óptica] está listo el anteojo cunado (5145_2015): [cuando] qu

F-3-A $\rightarrow$ M-2-A iera pasa

(5146_2015):

En el ejemplo 2, Juan continúa, a lo largo de la interacción, utilizando el tratamiento cortés de usted, mientras que Raitu mantiene en todos sus turnos una forma próxima y solidaria de tuteo voseante. Como se ha observado en nuestro corpus, a medida que los interlocutores intercambian mayor cantidad de mensaje, es probable que emerja el sistema de tuteo aunque en la oralidad se mantenga el ustedeo ${ }^{8}$. En el ejemplo 3, la presunción de que la forma "te olvide" corresponde a "te olvides" (es decir, caída de la s final) y no a "se olvide" (error de tipeo) se debe a la alta frecuencia de elisión de la s final en las formas desinenciales relevadas en el corpus SMS-CEBo9. Siguiendo este esquema, debemos suponer que en el ejemplo 4 se está tuteando a Román porque la forma "quiera pasá” no es congruente (la forma sería "quiera pase").

Por otra parte, dentro del sistema del español bonaerense, a pesar de ser un sistema mixto, el uso de las formas en tú no es habitual (Rigatuso, 2014). Es decir, su aparición en la interacción verbal contemporánea resulta un uso marcado. Sin embargo, en algunos casos aparecen formas verbales con o sin la presencia del pronombre tú explícito, como formas de expresividad afiliativa, con ciertas marcas de interdiscurso que remiten a canciones o frases hechas.

8 Esta observación se realizó a través de la inmersión etnográfica de la autora en diferentes ámbitos laborales de donde se extrajeron los datos y a partir de entrevistas complementarias que dieron cuenta del mantenimiento del ustedeo en las interacciones cara-a-cara.

9 Otros ejemplos de ello son:

(506) (M-1-B): "Todo piola q honda q conta? : -)"

(104) (F-1-A): "Aaaaaaa bueno! Pero pq se pelearon? Si quere conectate al eme asi no gastas crédito” (3094) (M-2-B) "Facu me pode yamar" 
Ejemplo 5:

Ámbito de uso: pareja. Contexto: una adolescente responde a un mensaje en el cual se le dicen groserías, preguntando si fue su pareja —dueño del teléfono- quien había enviado ese SMS.

F-I-A $\rightarrow$ M-1-A TU TU TU TU TU TU TU TU TIENES LA LLAVE DE MI CORAZÓN!!!! EMA TE AMO (734_2011): $\quad$ FUISTE VOS?

Ejemplo 6:

Ámbito de uso: pareja. Contexto: un joven envía un mensaje afectuoso a su novia.

M-2-A $\rightarrow$ F-2-A Buenas y santas mrss PUPITO! no me recordas q es lo q habias soñado? Porfis (2908_2008): $\quad$ Porfis!!! Dia nublado a punto de llover aca pero contento de saber que me quieres. Todo el dia estas conmigo, sos mi pensamiento (sentimiento) constante je BESO a la mas linda.

Ejemplo 7:

Ámbito de uso: relaciones sociales, vínculo entre amigas. Contexto: una joven envía un mensaje a una amiga, Pau, que está en la redacción final de sus tesis, para animarla.

F-2-A $\rightarrow$ F-2-A Tu si puedes! Tu si puedes! Tu si puedes! Tu si puedes =] no te desanimes. (4031_2015)

F-2-A $\rightarrow$ F-2-A Ay campeona, un msj al corazon :) Ahí vamos, a pasito d hormiga pero va (4032_2015)

F-2-A $\rightarrow$ F-2-A Escribí o llama cualquier cosa que necesites, de verdad... Pero lo tenés bastan(4033_2015) te cocinado. No te enriedes y estructuralo solamente =]

Ejemplo 8:

Ámbito de uso: relaciones sociales, vínculo amiga. Contexto: un joven quiere llamar a una amiga que vive en otra ciudad, y que hace mucho no ve, para saber cómo está.

M-2-A $\rightarrow$ F-2-A Mechita estas durmiendo la siesa o te puedo llamar?

(1783_2014)

F-2-A $\rightarrow$ F-2-A Llame nomas!

(1784_2014)

En el ejemplo 7 se observa, además, otro aspecto interesante: la posibilidad de percibir cierto cambio de código (del vos al tú10) en el comienzo del SMS 4018: “Escribí o llama”. El diccionario predictivo está codificado con las formas tuteantes, en general, y, salvo que el usuario incluyera la acentuación correspondiente a las formas imperativas o si la forma verbal del vos difiere de la del tú más allá de la acentuación (tal el caso de "escribí" vs. "escribe”),

10 Véase Rigatuso (2014). 
el resultado serán formas verbales que respondan, por defecto, al paradigma del tú. En el ejemplo 8, el cambio se da entre el vos y el usted. Caso contrario, será prominente la forma del tú dando como resultado esa alternancia de códigos en un mismo sintagma (además, posteriormente, aparece la forma "necesites" vs. "necesités"). Sin embargo, hay ejemplos que parecerían mostrar la confluencia de formas tuteantes y voseantes por parte del interlocutor (ejemplo 9).

Ejemplo 9:

Ámbito de uso: familiar. Contexto: Nélida escribe a su hija, que se está recuperando de una cesárea, para invitarla a que se acerque hasta su casa para ver a su sobrina ("Kari") y a su perro ("popi"). La hija está durmiendo y responde el yerno (“Carlos").

F-4-B $\rightarrow$ F-2-A Lau si no dormi s dile a Damián que te traiga desp [después]. Te lleva papa. (5230_2015) Ahora se acost[ó]. Estoy con Kari. Asi ves [vez] al popi.-

F-2-A $\rightarrow$ F-2-A Nelida recién se acuesta! Cuando se levante le digo. El lunes ira para bahia! (5231_2015)

La marcada preferencia por el trato de vos conlleva a pautas de uso donde este pronombre de confianza aparece explícitamente en contextos donde, en la interacción cara-a-cara, no se esperaría comprobar. En el siguiente pedido de empleo, una persona asimila el pronombre de confianza "vos" + el tratamiento ocupacional "ingeniero", siendo esta última expresión utilizada como forma nominal de tratamiento respetuoso. Esto es propio de la pauta innovadora verificada tempranamente (Rigatuso, 2000) y con un uso extendido en la actualidad (Rigatuso, 2014). El ejemplo 10 puede ser consecuencia de este cambio.

Ejemplo 10:

Ámbito de uso: laboral. Contexto: una persona desconocida envía un mensaje a un ingeniero solicitándole trabajo.

M- desconocido a M-2-A

(6150_2015)
Hola vos ingeniro [ingeniero] de la em presa [empresa] me dijiro [dijeron] que te hace falta gente -

Siguiendo con este modelo de formas desinenciales derivadas del pronombre de confianza vos, conjuntamente con tratamientos nominales de respeto, se comprueban también formas de "Señor-a + vos" (Rigatuso, 2000), "profesor-a + vos" (Rigatuso, 2014), "padre + vos" para los curas (Rigatuso, 2014), con el nombre de pila (ejemplo 11).

\section{Ejemplo 11:}

Ámbito de uso: laboral. Contexto: un delegado gremial consulta al empleador si sabe cuándo ingresará una persona a trabajar.

M-3-B a M-2-A. Y Yserñor [señor] laureano me averiguaste del ingreso de castillo cuanvo [cuan-

(6273_2015) do] a [va] a entrar 
Por último, un aspecto a considerar en cuanto a la variación vos/tú en la formas de confianza es que, si bien esta pauta de uso es mixta (Rigatuso, 2000), resulta importante destacar que, dado el mencionado uso del predictivo, es posible registrar formas verbales de tú que no son elecciones del emisor, tal como ocurre en el ejemplo 9, o por transferencia de frases hechas (ejemplos 5 y 7$)^{11}$.

Establecida la preferencia por el trato de vos en casi todas las díadas y en ámbitos de uso divergentes (como familiares y laborales), nos abocaremos en la próxima sección a analizar el papel central de las formas nominales vocativas y la función que cumplen en la comunicación por SMS.

\subsection{Las fórmulas nominales \\ 4.2.1. Distribución y variación de las formas vocativas}

El análisis cuantitativo de la aparición de formas vocativas en los SMS ha dado interesantes muestras de su alta frecuencia respecto a otros elementos catalizadores de la cortesía verbal. En su distribución, predomina el uso de formas nominales en función apelativa como término de llamado de atención respecto del conjunto de posibles formas apelativas, como interjecciones y las fórmulas de saludos. Así, en este estudio, nos hemos interesado por marcar las relaciones entre fórmulas de tratamiento vocativas como término de llamado de atención y formas de saludo iniciales, tales como hola, buen día, buenas tardes/noches y sus formas apocopadas y que revisten mecanismos de creación léxica como holis, güenas, etc.).

Tal como lo relevado en la preparación y apertura conversacional del español bonaerense (Rigatuso, 1987), la importancia de los elementos nominales en modo vocativo en estas instancias se constata dentro de SMS-CEBo: de los 6260 ${ }^{12}$ SMS analizados, el 33,5\% contiene una forma nominal en la preparación y apertura conversacional, de los cuales en el 6,6\% se combina saludo + fórmula de tratamiento nominal, y en el 0,2\%, vocativo apelativo + saludo. El tratamiento Che, por su parte, se comprueba solo en 0,3\% de los SMS. Un dato relevante es la escasa presencia de partículas o interjecciones que funcionan como términos de llamado de atención (0,7\%), entre las que destacan Ey y Eu.

En cuanto a la variación sociolingüística, según los datos obtenidos, se observa cómo el estilo del grupo socioeducativo bajo suele ser utilizar un registro formal y preferir formas

11 Si bien es cierto que no es una elección del emisor - ya que es el predictivo quien opta por la acentuación del paradigma del tú-, sería interesante ver lo que sucede desde la perspectiva del destinatario, ya que este lo que recibe es un trato de tú.

12 De la muestra total, se omitieron aquellos mensajes que habían sido producidos por empresas y aquellos de los cuales no se tenía información sobre el emisor. 
completas de saludo o saludo + vocativo como forma de preparación conversacional (véase ejemplo 8). Esto se verifica en lo que los valores disgregados indican: el grupo de hablantes de nivel socioeducativo bajo utiliza saludo + vocativo un 13,8\% de las veces y saludo solo $6,7 \%$, mientras que los interlocutores del grupo alto lo hacen en un 5,8\% para el saludo + vocativo y $3,1 \%$ para saludo solo. Es decir, el grupo socioeducativo bajo duplica la cantidad de veces que opta por formas de saludo (con o sin vocativo). En la tabla I se resume la distribución de las fórmulas de tratamiento nominales vocativas dentro del conjunto de formas de apelación consideradas.

\section{TABLA 1}

Distribución de las formas vocativas respecto de otras formas de apelación en el corpus SMS-CEBo

\begin{tabular}{c|c|c|c|c|c|c}
$\begin{array}{c}\text { NIVEL } \\
\text { SOCIO- } \\
\text { EDUCATIVO }\end{array}$ & $\begin{array}{c}\text { AUSENCIA } \\
\text { DE } \\
\text { SALUDOSY } \\
\text { VOCATIVOS }\end{array}$ & $\begin{array}{c}\text { FORMA NOMINAL } \\
\text { COMO TÉRMINO } \\
\text { DE LLAMADO } \\
\text { DE ATENCIÓN }\end{array}$ & $\begin{array}{c}\text { VOCATIVO } \\
\text { + SALUDO }\end{array}$ & $\begin{array}{c}\text { SALUDO + } \\
\text { VOCATIVO }\end{array}$ & SALUDO & INTERJECCIÓN \\
\hline Bajo & $49.5 \%$ & $29.7 \%$ & $0.2 \%$ & $13.8 \%$ & $6.7 \%$ & $0.3 \%$ \\
\hline Alto & $63.7 \%$ & $26.5 \%$ & $0.2 \%$ & $5.8 \%$ & $3.1 \%$ & $0.8 \%$ \\
\hline
\end{tabular}

Asimismo, según el grupo etario al que pertenezcan los hablantes, tendremos una distribución diferente de la aparición de elementos apelativos. Así, entre los adolescentes y jóvenes prácticamente se duplican las formas nominales vocativas, así como el uso de interjecciones como términos de llamado de atención, y su acumulación discursiva se configura como una estrategia de mantenimiento de las relaciones sociales dentro de este grupo. Su ausencia puede derivar en conflictos comunicativos, en particular en la díada de pareja (Cantamutto, 2017). Por otra parte, el conjunto de formas de apertura general (con o sin formas vocativas) tiene gran incidencia en los grupos etarios de adultos (3 y 4) y adultos mayores (5).

\section{TABLA 2}

Distribución de formas vocativas según grupo etario en el corpus SMS-CEBo

\begin{tabular}{c|c|c|c|c|c|c} 
GRUPO & $\begin{array}{c}\text { AUSENCIA DE } \\
\text { SALUDOS Y } \\
\text { VOCATIVOS }\end{array}$ & $\begin{array}{c}\text { FORMA NOMINAL } \\
\text { COMO TÉRMINO } \\
\text { DE LLAMADO } \\
\text { DE ATENCIÓN }\end{array}$ & $\begin{array}{c}\text { VOCATIVO } \\
\text { + SALUDO }\end{array}$ & $\begin{array}{c}\text { SALUDO + } \\
\text { VOCATIVO }\end{array}$ & SALUDO & $\begin{array}{c}\text { PARTICULAS O } \\
\text { INTERJECCIONES }\end{array}$ \\
\hline 1 & $68.4 \%$ & $25.8 \%$ & $2.2 \%$ & $1.5 \%$ & $2.1 \%$ \\
\hline 2 & $59.3 \%$ & $30.3 \%$ & $0.3 \%$ & $6.3 \%$ & $3.2 \%$ & $0.6 \%$ \\
\hline 3 & $62.2 \%$ & $17.9 \%$ & & $13.7 \%$ & $6.3 \%$ & $5.1 \%$ \\
\hline 5 & $69.7 \%$ & $16.8 \%$ & $3.2 \%$ & $3.2 \%$ \\
\hline
\end{tabular}


Algo similar ocurre cuando se analiza la variación según sexo. En este caso se observa poca diferencia respecto al sexo de quien escribe el SMS, con una leve tendencia a la aparición de estas formas en las mujeres. En la tabla 3 se refleja cómo hay un leve aumento de la presencia de vocativos y formas de saludo + vocativo en los SMS de mujeres. Sin embargo, la preferencia por interjecciones como términos de llamado de atención es casi el doble en los hombres respecto a las mujeres.

\section{TABLA 3}

Distribución de formas vocativas según sexo del emisor en SMS-CEBo

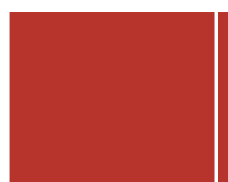

AUSENCIA DE

SALUDOSY

VOCATIVOS

FORMA NOMINAL COMO TÉRMINO

DE LLAMADO

DE ATENCIÓN
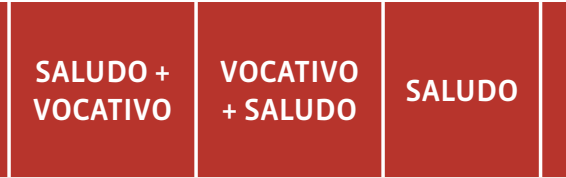

INTERJECCIÓN

\begin{tabular}{lllllll} 
femenino & $59.7 \%$ & $28.1 \%$ & $7.8 \%$ & $0.2 \%$ & $3.6 \%$ & $0.5 \%$ \\
\hline masculino & $64.6 \%$ & $25.4 \%$ & $5.6 \%$ & $0.2 \%$ & $3.3 \%$ & $0.9 \%$ \\
\hline
\end{tabular}

El análisis de los datos según el dominio de uso en el cual ocurren las interacciones por SMS arroja luz sobre interesante fenómenos de variación. Tal como observamos en la tabla 4, dentro del ámbito de las relaciones sociales, los intercambios entre parejas constan de formas nominales e interjecciones como formas de apelación en casi la mitad de los intercambios y, en la mayoría de los casos, se observa una acumulación de formas destinadas al interlocutor. Otro aspecto que debemos señalar es el aumento de saludos, propio del menor grado de cercanía, sin el acompañamiento de formas vocativas, dentro del ámbito laboral en relación a díadas pertenecientes al ámbito familiar o de pareja. En estas relaciones, los saludos con vocativo (en posición inicial o integrando la forma de saludo), solo representan el 3,9\% entre amigos y $1,3 \%$ en parejas, mientras que para los vínculos dentro del dominio laboral la cifra alcanza el $11,8 \%$ de los casos de SMS-CEBo.

\section{TABLA 4}

Distribución del vocativo según ámbito de uso

\begin{tabular}{c|c|c|cc} 
& AMISTAD & FAMILIAR & LABORAL & PAREJA \\
\hline Ausencia de vocativos y saludos & $61,6 \%$ & $68,0 \%$ & $58,2 \%$ & $56,8 \%$ \\
\hline Presencia de vocativo (con/sin saludo) & $36,6 \%$ & $28,3 \%$ & $35,2 \%$ & $41,9 \%$ \\
\hline Presencia de saludo (sin vocativo) & $1,8 \%$ & $3,7 \%$ & $6,6 \%$ & $1,3 \%$ \\
\hline
\end{tabular}

Es interesante señalar que se reproduce, en cierta forma, la lógica de las estrategias de cortesía que parecen ser menos frecuentes en relaciones de mucha confianza (Calsamiglia Blan- 
cafort y Tusón Valls, 2002: 163), como las familiares, y que tienen predominio en las relaciones más conflictivas, como las laborales y en la relación de pareja, con mayor carga de afectividad.

Por último, atendiendo a que las diferentes formas que cumplen una función apelativa dentro del SMS se direccionan, precisamente, hacia el interlocutor, hemos realizado análisis orientados a la influencia que tienen las características del interlocutor en la presencia o no de formas apelativas. Siguiendo una de las hipótesis de nuestra investigación, según el interlocutor deberían imprimirse huellas de las estrategias empleadas por parte del hablante para poder alcanzar sus metas concretas (Zimmerman, 2005). Sin embargo, los resultados señalan que la variable sexo del destinatario influye en el fenómeno estudiado así como el nivel socioeducativo del interlocutor. En la tabla 5 se puede observar cómo aumenta el porcentaje de vocativos según el sexo del destinatario: no se registran variaciones significativas salvo en el aumento de la coocurrencia de fórmulas de saludos y formas vocativas en los mensajes enviados de mujeres a hombres (10.31\%), un uso levemente inferior de vocativos en los mensajes producidos por hablantes masculinos hacia hombres (22.46\%) que hacia mujeres (32.40\%).

\section{TABLA 5}

Variación de las formas vocativas según sexo del destinatario

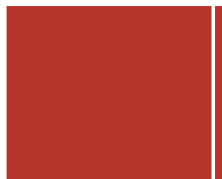

AUSENCIA DE

VOCATIVOS

O SALUDOS

FORMA NOMINAL COMO TÉRMINO

DE LLAMADO

DE ATENCIÓN
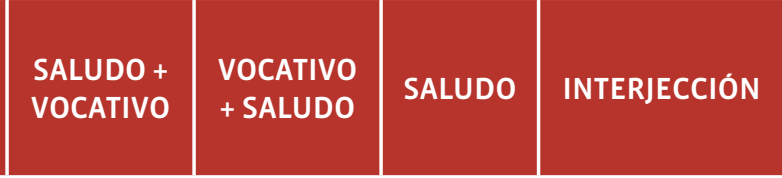

$\mathrm{FaF}$

\begin{tabular}{|c|c|c|c|c|c|c|}
\hline $\begin{array}{c}F \text { a F } \\
(m=2051)\end{array}$ & $60.95 \%$ & $27.99 \%$ & $6.48 \%$ & $0.10 \%$ & $3.71 \%$ & $0.78 \%$ \\
\hline $\begin{array}{c}M \text { a M } \\
(m=2257)\end{array}$ & $66.55 \%$ & $22.46 \%$ & $5.49 \%$ & $0.35 \%$ & $4.12 \%$ & $1.02 \%$ \\
\hline $\begin{array}{c}M \text { a F } \\
(m=963)\end{array}$ & $59.81 \%$ & $32.40 \%$ & $5.92 \%$ & & $1.25 \%$ & $0.62 \%$ \\
\hline $\begin{array}{c}F a M \\
(m=989)\end{array}$ & $57.33 \%$ & $28.61 \%$ & $10.31 \%$ & $0.40 \%$ & $3.34 \%$ & $0.00 \%$ \\
\hline
\end{tabular}

Por otra parte, los datos relevados según el grupo socioeducativo del destinatario, la presencia de vocativos en los mensajes dirigidos a hablantes del grupo alto es mayor ( $28 \%$ vs. 20\% al bajo) mientras que el saludo (con y sin formas vocativas) prevale en los mensajes enviados a hablantes del grupo socioeducativo bajo (14\% vs. 10\%).

\subsubsection{Descripción de las formas nominales}

En anteriores estudios nos hemos detenido en la descripción de las formas nominales con función vocativa relevadas con mayor frecuencia (Cantamutto, 2014, 2015a, 2016, 2017). En to- 
dos los casos, se ha observado la importancia de la díada en la cual se inscriben estos elementos y que dan cuenta del valor que adquieren estas formas nominales en los grupos etarios de adolescentes y jóvenes, que en el caso de los hombres tiende, además, a la preferencia por formas anticorteses (Zimmerman, 2002).

Dentro del ámbito de las relaciones sociales, es interesante atender a cómo estos ítems léxicos son portadores de muchos de los mecanismos de acortamiento y de creación léxica que se observan en la comunicación por SMS. A continuación, en la tabla 6, ilustramos con algunos ejemplos prototípicos siguiendo la propuesta de Rigatuso (1994).

\section{TABLA 6}

Fórmulas de tratamiento nominales utilizadas por hablantes del español bonaerense
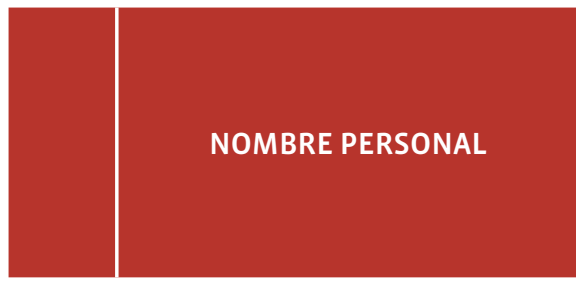

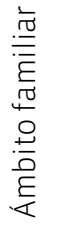

(padre $\rightarrow$ hijo): nombre de pila

(hijo $\rightarrow$ padre): sobrenombre (entre hermanos / primos): sobrenombre y nombre apocopado

\section{TÉRMINOS MARCADORES \\ DE RELACIÓN (TÉRMINOS DE PARENTESCO, \\ TÉRMINOS DE AMISTAD Y CORDIALIDAD, TRATAMIENTOS OCUPACIONALES)}

(padre $\rightarrow$ hijo): hijo-a, hijito (hijo $\rightarrow$ padre): mamá, papá, padre, madre, papi, mami y sus formas apocopadas. (entre primos): prima

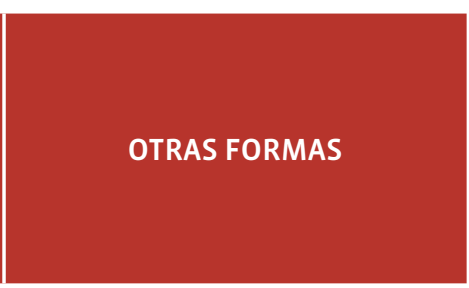

(hijo $\rightarrow$ padre) Usos metafóricos afectuosos o extensión de significados que meliorizan en su función el significado (uso ficticio) ${ }^{13}$ : viejo-a.

(amigos): insultos ficticios (gay, pene, yegua/o, boluda/bolas, crota, enana, goma, amigato).

(amigos): amigo-a, amigis y ami. (amigos): formas ameliorativas (pareja): extensiones semánticas de distintas formas léxicas hermosa, corazón, amor, beba, bonito, mi vida, princesita.

(empleado $\rightarrow$ empleador): Tratamientos ocupacionales ingeniero/a, arquitecto, profesor/a y profe, seño y Tratamientos sociales de índole general: don, señora, señor. (empleador $\rightarrow$ empleado): señora, señor 


\section{Conclusiones}

En estas páginas hemos ofrecido un panorama de las fórmulas de tratamiento en la comunicación por SMS en español bonaerense. En primer lugar, nos detuvimos a identificar el sistema de fórmulas de tratamiento que orienta los intercambios, siendo el preferido el trato de confianza de vos en la mayoría de los intercambios, en todos los dominios de uso, a excepción de la presencia de formas en usted en intercambios entre poco conocidos o cuando la relación jerárquica es asimétrica. Asimismo, no se detectó variación significativa en estos usos respecto a otras variables sociolingüísticas. Esto se debe a que, en la mayoría de los intercambios que forman parte de SMS-CEBo, IOs usos relevados corresponden a la cortesía informal que establece una comunicación en relaciones solidarias (Brown y Gilman, 1960), en las cuales se utiliza el pronombre de confianza vos.

El estudio de los vocativos resulta particularmente interesante. En primer lugar, la mayoría de los intercambios se dan entre conocidos, y se envía a un único destinatario, por lo que no es necesario seleccionar entre posibles interlocutores. Los interactuantes se conocen y, por imposiciones propias del medio, presuponen que el SMS enviado está direccionado a un único destinatario. Podría decirse que el uso marcado del vocativo en estas interacciones corresponde a una búsqueda por focalizar en el destinatario, realizado con diferentes formas nominales (Rigatuso, 2009).

Dentro del ámbito de las relaciones sociales, el corpus ofrece datos respecto a los tratamientos utilizados por amigos mayoritariamente dentro del grupo de adolescentes y jóvenes. En dichos grupos, aparecen formas similares en el intercambio de mensajes entre varones que, además del uso de los nombres de pila y sobrenombres, responden a elementos anticorteses (gay, pija y cambios al género femenino, como amiga, entre hombres). Entre mujeres, predomina, además de los nombres y sobrenombres, el uso de gorda, amiga y otras formas como linda o hermosa. En los grupos de adultos (3 y 4) y adultos mayores (5) se verifican mayormente el uso de los nombres de pila y la preferencia por formas que den cuenta de las relaciones entre los interactuantes. Este fenómeno se da también en el ámbito familiar y laboral.

El estudio cuantitativo de la variación en el uso de vocativos ha demostrado, por un lado, la importancia de este elemento en la comunicación por SMS. En promedio, el 34,3\% de los mensajes de SMS-CEBo contiene un tratamiento en función vocativa. Ese número se incrementa si solo se consideran los mensajes iniciativos, y se descartan aquellos mensajes de cierre de una interacción por SMS o de confirmación de recepción, cuya estructura suele ser de una sola palabra (gracias, ok y dale).

Dentro de los recursos de expresividad que se verifican en la comunicación por SMS (Cantamutto, 2016), el vocativo cumple una función estructurante en la interacción. No solo en las fases de preparación (en muchos casos, acompañado de las fórmulas de saludo) sino en los 
distintos mensajes permite focalizar en el interlocutor y direccionar hacia él algún segmento de los mismos. La falta de recursos paralingüísticos favorece el uso de este fenómeno como elemento focalizador.

A modo de cierre, recuperamos la importancia del dominio de uso como elemento que condiciona la presencia de fórmulas vocativas a lo largo de todo el intercambio. Si bien una mirada cualitativa nos ofrecería mucha divergencia respecto a la riqueza de los vocativos en el ámbito de las relaciones sociales (amistad y pareja) respecto al ámbito laboral, la cortesía estratégica se activa en el uso de vocativos y fórmulas de saludo en las díadas más conflictivas. En tal sentido, en futuras investigaciones se intentará describir cualitativamente las formas nominales utilizadas en cada uno de estos ámbitos así como la confluencia de otros fenómenos de expresividad que resultan pertinentes para los SMS.

\section{Bibliografía citada}

Alcantara-Pla, Manuel, 2014: "Las unidades discursivas en los mensajes instantáneos de wasap”, Estudios de Lingüística del Español 35, 223-242.

Alonso-Cortés, Ángel 1999: "Las construcciones exclamativas. La interjección y las expresiones vocativas" en Ignacio Bosque Muñoz y Violeta Demonte Barreto (dirs.): Gramática Descriptiva de La Lengua Española, vol. III, Madrid: Espasa, 3993-4050.

Álvarez Gonzaga, Braulio, 2010: “¿Es el lenguaje SMS un deterioro del idioma?” [disponible en http://braulioedunet.webcindario.com/ensayo-sms.pdf].

Avedaño, Virginia, 2007: “El lenguaje del chat los SMS: ¿un nuevo género discursivo?”, Portal Educar [disponible en http://portal.educ.ar/debates/eid/lengua/debate/como-influyen-elchat-y-las-nu.php].

Bernicot, Josie, Olga Volckaert-Legrier, Antonine Goumi y Alain Bert-Erboul, 2012: "Forms and functions of SMS messages: A study of variations in a corpus written by adolescents", Journal of Pragmatics 44 (12), 1701-1715 [disponible en http://doi.org/10.1016/j.pragma.2012.07.009].

Bravo, Diana, 2001: "Sobre la cortesía lingüística, estratégica y conversacional en español", Oralia: Análisis del Discurso Oral 4, 155-184.

BRIz, Antonio, 2004: "Cortesía verbal codificada y cortesía verbal interpretada en la conversación” en Diana Bravo y Antonio Briz (coords.): Pragmática sociocultural: estudios sobre el discurso de cortesía en español, Barcelona: Ariel, 67-93.

Brown, Roger, y Albert GiLman, 1960: "The pronouns of power and solidarity” en Thomas Sebeok (ed.): Style in Language, Cambridge: MIT press, 253-276. 
Brown, Penelope, y Stephen Levinson, 1987: Politeness. Some universals in language usage, Cambridge: Cambridge University Press.

Bucher, Claudia, 2015: "Code-switching in SMS communication” en Eivin Torgersen, Stian HÅrsTAD, Brit Mehlum y Unn Rørneland (eds.): Language Variation-European Perspectives V: Selected papers from the Seventh International Conference on Language Variation in Europe (ICLaVE 7), Amsterdam: John Benjamins publishing, 43-54.

Calsamiglia Blancafort, Helena, y Amparo Tusón Valls, 2002: Las cosas del decir. Manual de análisis del discurso, Barcelona: Ariel.

Calero Vaquera, María Luisa, 2014: "El discurso del WhatsApp: entre el Messenger y el el SMS", Oralia 17, 85-114.

Cantamutto, Lucía, 2012: El uso de los mensajes de texto en el habla adolescente del español bonaerense. Tesis de Licenciatura, Bahía Blanca: Universidad Nacional del Sur [disponible en http://repositoriodigital.uns.edu.ar/handle/123456789/2852].

Cantamutto, Lucía, 2013a: "La conformación de un corpus de mensajería de texto: Ia interacción verbal mediada digitalmente" en Lidia Gambón (ed.): IV Jornadas de Investigación en Humanidades. Homenaje a Laura Laiseca. Ebook, Bahía Blanca: Universidad Nacional del Sur, 97-106.

Cantamutto, Lucía, 2013b: "La recursividad de las interacciones contemporáneas. Límites teórico-metodológicos del estudio de los SMS como conversación”, Revista de Ciencias Sociales de la Universidad Nacional de Quilmes "Al abordaje de la comunicación contemporánea. Sociedad y cultura en los mundos de la mediación digital" 23, 83-104.

Cantamutto, Lucía, 2014: "El discurso de los mensajes de texto en el habla adolescente del español bonaerense" en Alejandro PARIn y Mabel GIAmmatteo (eds.): Lenguaje, discurso e interacción en los espacios virtuales, Mendoza: Editorial FFyL-UNCuyo / SAL, 65-81.

Cantamutto, Lucía, 2015a: "Aspectos pragmáticos de la literacidad digital: la gestión interrelacional en la comunicación por teléfono móvil”, Revista Internacional de Tecnología, Conocimiento y Sociedad 5 (1), 95-111.

Cantamutto, Lucía, 2015b: “Ok. Cortado de mierda:\$'. Las fórmulas de tratamiento como recurso expresivo" en Sara Pérez (coord.): Actas del VI Coloquio de Investigadores en Estudios del Discurso. III Jornadas Internacionales sobre Discurso e Interdisciplina, Bernal: Universidad Nacional de Quilmes.

Cantamutto, Lucía, 2016: “La comunicación por mensajes de texto en el español bonaerense: uso y percepción” en Gimena del Rio Riande, Lucía Cantamutto y Gabriela Striker, Gabriela (ed.): Las Humanidades Digitales desde Argentina. Tecnologías, Culturas, Saberes, Buenos Aires: FyLUBA, 305-326. 
Cantamutto, Lucía, 2017: “Economía, claridad y expresividad lingüísticas: el estilo comunicativo digital del teléfono móvil en el español bonaerense" en Mabel Giammatteo, Patricia Gubitosı y Alejandro Parinı (eds.): El español en la red. usos y géneros de la comunicación mediada por computadora, Madrid: Iberoamericana Vervuert, 93-119.

Cantamutto, Lucía, y Cristina Vela Delfa. 2016: "El discurso digital como objeto de estudio: de la descripción de interfaces a la definición de propiedades." Aposta. Revista de Ciencias Sociales 69, 296-323.

Cougnon, Louise-Amélie, 2015: Langage et SMS. Une étude internationale des pratiques actueIles, Louvain : Presees universitaires de Louvain.

Cougnon, Louise-Amélie, y Cédrick FaIRon 2012: "Introduction”. Lingvisticae Investigationes 2, 35, $155-162$.

Dı Tulııo, Ángela, 2010: Manual de gramática española, Buenos Aires: Waldhuter Editores.

Galán Rodriguez, Carmen 2002: "En los arrabales de la comunicación: Ios mensajes SMS”, Anuario de Estudios Filológicos 25, 103-117.

Galán Rodriguez, Carmen 2003: "SMS: ¿un nuevo modelo de lenguaje?", Español Actual 76, 93-104.

Ghezzi, Maddalena, y María Sampedro Mella, 2015: "Influencia de la variable nivel sociocultural en el uso de las formas de tratamiento", Pragmalingüística 23, 61-78.

Herring, Susan, 1996: "Two Variants of an Electronic Message Schema” en Susan Herring (ed.): Computer Mediated Communication. Linguistic, Social and Cross-Cultural Perspective, Amsterdam: John Benjamins publishing, 81-106.

Hummel, Martin, Bettina Kluge y María Eugenia Vázuez, 2010: Formas y fórmulas de tratamiento en el mundo hispánico, México: El colegio de México.

Kaul de Marlangeon, Silvia, 2008: "Tipología del comportamiento verbal descortés en español" en Antonio Briz (ed.): Actas del III Coloquio Internacional del Programa EDICE, Valencia: Universidad de Valencia, 254-266.

Kerbrat-Orecchioni, Catherine, 1996: La conversation, Paris: Seuil.

LeECH, Geoffrey, 1999: "The distribution and functions of vocatives" en Hilde Hasselgärd y Signe OkSEFJELL (eds.): Out of corpora, Amsterdam: Rodopi, 107-118.

López Alonso, Covadonga, 2006: "El correo electrónico" en Covadonga López Alonso y Arlette Séré (eds.): Estudios de Lingüística del Español (24) [disponible en https://ddd.uab.cat/record/53648]. 
MARIS, Claire Lobet, y Laurent HenIN, 2002: "Hablar sin comunicar o comunicar sin hablar: del GSM al SMS”, Estudios de Juventud (57), 101-114.

Martín Gascueña, Rosa, 2016: “La conversación guasap”, Pragmática Sociocultural 4 (1), 108-134.

Morel, Etienne, Claudia Bucher, Simona Pekarek Doehler y Beat SiebenhaAr, 2012: "SMS communication as plurilingual communication: Hybrid language use as a challenge for classical codeswitching categories", Lingvisticae Investigationes 35 (2), 260-288 [disponible en http://doi. org/10.1075/li.35.2.08mor].

NeuAge, Terell, 2005: Conversational Analysis of chatroom. Tesis doctoral, Adelaida: University of South Australia.

Noblía, María Valentina, 1999: "Conversación y Comunidad. Las chats en la comunidad virtual”, Revista Iberoamericana de Discurso y Sociedad 2 (1), 77-99

Paredes Duarte, María Jesús, 2008: “El principio de economía lingüística”, Pragmalingüística 1516, 166-78.

PANCKHURST, Rachel, 2009: "Short Message Service (SMS): typologie et problemátiques futures" en Teddy Arnavielle (coord.): Polyphonies, pour Michelle Lanvin, Montpellier: Université PaulValéry Montepellier 3, 33-52.

Rigatuso, Elizabeth, 1987: "Dinámica de los tratamientos en la interacción verbal: preparación y apertura conversacionales", Anuario de Lingüística Hispánica III, 161-182.

Rigatuso, Elizabeth, 1992: Lengua, historia y sociedad. Evolución de las fórmulas de tratamiento en español bonaerense (1830-1930), Bahía Blanca: Universidad Nacional del Sur.

Rigatuso, Elizabeth, 1994: Fórmulas de tratamiento y familia en español bonaerense actual. Tesis doctoral, Bahía Blanca: Universidad Nacional del Sur.

Rigatuso, Elizabeth, 2000: “Señora (...) ¿No tenés más chico?’ Un aspecto de la pragmática de las fórmulas de tratamiento en español bonaerense", Revista Argentina de Lingüística 16, 293-344.

Rigatuso, Elizabeth, 2003: "Cortesía, tratamientos e identidad cultural en encuentros de servicio en español bonaerense" en Actas II Jornadas interdisciplinarias del sudoeste bonaerense, Bahía Blanca: Universidad Nacional del Sur, 157-179.

Rigatuso, Elizabeth, 2007: ““iChe, vos pibe!’ Usos y valores comunicativos del vocativo en español bonaerense actual" en Nidia Burgos y Elizabeth Rıgatuso (eds.): La modernización del sudoeste bonaerense. Reflexiones y polémicas en el ámbito educativo, lingüístico y literario, Bahía Blanca: Archivo de la Memoria-Secretaría de Comunicación y Cultura, Universidad Nacional del Sur, 81-93. 
Rigatuso, Elizabeth, 2008: “iQué! ¿Tienen calor?” Conversación de contacto en español bonaerense: de interacciones institucionales, de servicio y sociales", Oralia: Análisis del Discurso Oral 11, 133-168.

Rigatuso, Elizabeth, 2009: “'Madryn, pasame el apunte’. Aspectos léxico-semánticos del vocativo en el español bonaerense actual" en Mabel Cernadas de Bulnes y José Marcilese (eds.): Política, Sociedad y Cultura en el Sudoeste Bonaerense, Actas de las V Jornadas Interdisciplinarias del Sudoeste Bonaerense, Bahía Blanca: EdiUns, 369-386.

Rigatuso, Elizabeth, 2014: "Cuestiones de variación lingüística en un sistema de tratamientos del español de la Argentina. El español bonaerense 1 : extensiones metafóricas de términos de parentesco filiales", Boletín de La Academia Argentina de Letras LXXI, 87-150.

Spencer-OAtey, Helen, 2000: "Rapport Management: A Framework for Analysis" en Helen SpencerOATEY (ed.): Culturally Speaking: Managing Rapport through Talk across Cultures, London / New York: Continuum, 11-46.

TAGG, Caroline, 2009: A corpus linguistics study of SMS text messaging. Tesis doctoral, Birmingham: University of Birmingham.

TJORA, Aksel, 2011: "Invisible whispers: accounts of SMS communication in shared physical space”, Convergence: The International Journal of Research into New Media Technologies 17, 193211 [disponible en http://doi.org/10.1177/1354856510394604].

Van Dıık, Teun A. 2000: El discurso como interacción social, Barcelona: Gedisa.

Vela Delfa, Cristina, 2005: El correo electrónico. El nacimiento de un nuevo género. Tesis doctoral, Madrid: Universidad Complutense de Madrid.

Vela Delfa, Cristina, 2014: "Las interacciones conversacionales escritas: aproximación a una tipologia del género chat”, Quaderns Digita/s 1-02-2014 [disponible en http://www.quadernsdigitals.net/index.php?accionMenu=hemeroteca.VisualizaArticulolU.visualiza\&articulo_id=1 1292\&PHPSESSID=e227785db815e39900ec3beg4659d39b].

Vela Delfa, Cristina, y Juan Jímenez Gómez 2011: "El sistema de alternancia de turnos en los intercambios sincrónicos mediatizados por ordenador”, Pragmalingüística 19, 121-138.

Vela Delfa, Cristina, y Lucía Cantamutto, 2015a: "Methodological approach to the design of digital discourse corpora in Spanish. Proposal of the CoDiCE Project", Procedia-Social and Behavioral Sciences 198, 494-499.

Vela Delfa, Cristina, y Lucía Cantamutto, 2015b: “Problemas de recogida y fijación de muestras del discurso digital", CHIMERA. Romance Corpora and Linguistic Studies 2, 131-155.

Verschueren, Jef, 2002: Para entender la Pragmática, Madrid: Gredos. 
Xue, Nianwen, Qishen Su y Sooyoung Jeong, 2016: "Annotating the discourse and dialogue structure of SMS message conversations" en Law X - The 1oth Linguistic Annotation Workshop, Berlin: Association for Computational Linguistics, 180-187.

Yus, Francisco, 2010: Ciberpragmática 2.0. Nuevos usos del lenguaje en internet, Ariel: Barcelona.

Yus, Francisco, 2016: “Towards a Cyberpragmatics of Mobile Instant Messaging”, en Jesús Romero Trillo (ed.): Yearbook of Corpus Linguistics and Pragmatics, Berlín: Springer International Publish, 7-26.

Zimmerman, Klaus, 2002: “La variedad juvenil y la interacción verbal entre jóvenes” en Félix RoDRíguez (ed.): El lenguaje de los jóvenes, Barcelona: Ariel, 137-161.

Zimmerman, Klaus, 2005: “Construcción de la identidad y anticortesía” en Diana Bravo (ed.): Estudios de la (des)cortesía en español. Categorías conceptuales y aplicaciones a corporas orales y escritos, Estocolmo: Universidad de Estocolmo, 245-272. 